\title{
Diagnostic and Therapeutic Challenges
}

\author{
Dr. Luiz H. Lima, MD ${ }^{\star}$, Dr. Eric Shakin, MD $\dagger$, Dr. N. Nida Sen, MD, MHSc ${ }^{\ddagger}$, Dr. Robert B. \\ Nussenblatt, MD $\ddagger$, and Dr. Lawrence A. Yannuzzi, MD \\ * LuEsther T. Mertz Retinal Research Center, Manhattan Eye, Ear, and Throat Hospital, New \\ York, New York † Department of Ophthalmology, New York University, New York, New York ‡ \\ Department of Uveitis and Ocular Immunology, National Eye Institute, National Institutes of \\ Health, Bethesda, Maryland
}

\section{Case Report}

A 53-year-old white woman first presented with flashes and floaters in 1990. There was no history of significant medical problems, and she was taking no medications. With regard to her ocular status, she had been a glaucoma suspect, but her intraocular pressures, visual fields, and optic nerve could not confirm the diagnosis of glaucoma. When first examined, her comprehensive ophthalmologist noted only a mild degree of optic disk swelling with a $20 / 20$ visual acuity bilaterally. She was evaluated neuro-ophthalmologically on suspicion of papilledema, and her computed tomographic scan of the brain and cerebrospinal fluid analysis were normal. A preliminary diagnosis of pseudotumor cerebri was made, and she was treated with oral acetazolamide without clinically evident improvement. At this time, a medical evaluation showed only a mild degree of systemic hypertension, and she was placed on Prinivil (5 mg) twice a day. Her medical examination included the standard investigation for intermediate uveitis, including granulomatous disease. The findings of her chest $x$-ray and a conjunctival biopsy, in search of evidence for possible sarcoidosis, were normal. She did have a positive HLA-A29 antibody. At that point, she was referred to the National Eye Institute for consultation regarding a mild uveitic infiltration in the vitreous in conjunction with multiple depigmented spots in the fundus.

The examination at the National Eye Institute showed best-corrected acuity of 20/60 in the right eye and 20/25 in the left eye. The positive findings were limited to the posterior segment where there was mild vitreous cellular infiltration, cystoid macular edema, optic nerve edema, and numerous subretinal, pale, cream-colored lesions (Figure 1). There was also mild phlebitis evident on fluorescein angiography, a few perivascular hemorrhages, and some peripheral retinal vascular sheathing (Figure 1). The diagnosis of birdshot chorioretinopathy was made, and the patient was treated with sub-Tenon injections of triamcinolone acetonide.

During the ensuing 13 years, 2 retinal specialists continued to manage the patient locally. Exacerbations or emissions of the inflammatory changes in the vitreous, progressive optic atrophy, and diffuse retinal vascular inflammation and sheathing were evident. In 2000, she was noted to have deposits of lipid deposition in association with retinal arteriolar

RETINA $^{\circledR}$, The Journal of Retinal and Vitreous Diseases, encourages readers to submit Diagnostic and Therapeutic Challenges to retina@retinajournal.com. Cases for the Diagnostic and Therapeutic Challenges section should include a detailed history of the patient, the diagnosis, the workup, the management, and finally, the question or questions that the submitter wishes to have answered by the consultants. 
macroaneurysm formation in both the eyes. A few of these macroaneurysms have been treated with laser photocoagulation because of persistent and progressive lipid exudation threatening her visual function according to the treating retinal specialist.

In summary, this patient presented initially in her fifth decade with manifestations, which were clearly viewed as birdshot chorioretinitis with multiple pale, ovoid lesions throughout the fundus, disk swelling, macular edema, and retinal vascular inflammatory changes. A positive HLA-29 determination was consistent with that diagnosis, and there was no evidence of any other systemic disease, except for a mild degree of systemic hypertension. Later, she developed multiple arteriolar macroaneurysms with lipid exudation in association with the retinal vascular changes and neuroretinitis. This case is presented for discussion of diagnosis.

We asked several experts for their opinion.

\section{Dr. Lee M. Jampol (Chicago, Illinois)}

The patient described in this scenario presents a clinical challenge. There are characteristics of what seem to be two distinct processes. The first finding is the occurrence of deep retinal or choroidal lesions scattered in the posterior poles. These lesions have developed in association with a long history of inflammation, including disk edema, cystoid macular edema, and retinal vascular leakage. The second finding is the occurrence of opacification of retinal arterioles with the development of multiple macroaneurysms.

Arterial macroaneurysms may be seen with hypertension or arteriosclerosis, which may be single or multiple. Occasionally, they occur postembolic. This patient has multiple lesions not consistent with these diagnoses. Arterial macroaneurysms have also been reported in association with Coats disease, Leber disease, and retinal arterial and venous occlusions. There is one case report describing the association of macroaneurysms with polypoidal choroidal vasculopathy.

In younger patients, an important cause of retinal arterial aneurysms, particularly when multiple and bilateral, is idiopathic retinal vasculitis, aneurysms, and neuroretinitis (IRVAN). ${ }^{1}$ Patients with IRVAN have evidence of vasculitis; the aneurysms may occur on or near the optic nerve. The patients often develop capillary nonperfusion, especially in the peripheral retina, and retinal neovascularization. They often have lipid exudation in proximity to the aneurysms and in the macula. They may have macular thickening.

It is uncertain whether this patient has IRVAN. We do not have evidence in the case material presented of peripheral nonperfusion or retinal neovascularization. There is no specific testing for IRVAN, and our patients' findings would be consistent with but not diagnostic of IRVAN.

Birdshot chorioretinopathy is seen in young to middle-aged patients who develop vitreal and retinal inflammation. ${ }^{2}$ Birdshot lesions are scattered around the posterior pole. Disk swelling and leakage, macular swelling and leakage, arterial narrowing, and ultimately diminished electroretinography and nyctalopia are seen. The lesions in this patient would be consistent with birdshot, and the presence of HLA-A29 is certainly suggestive of birdshot. It is possible that chronic inflammation from birdshot induced the macroaneurysms, but we have not seen this previously. More than 95\% of patients with birdshot have HLA-A29, but it should be pointed out that $7 \%$ of the normal population has this. Thus, the occurrence of HLA-A29 could be coincidental. If HLA-A29 is a red herring, what other disease could this be? Sarcoidosis can present with multiple midperipheral and peripheral chorioretinal lesions. 3,4 They often inferiorly occur (we do not know whether this describes this patient). The 
lesions may progress to chorioretinal scars. Vitreous organization can be seen. Similarly, some patients have "presumed" sarcoidosis, similar to sarcoidosis but without definite systemic evidence of sarcoid. Patients with multifocal choroiditis (MFC) of this type (including sarcoid and presumed sarcoid) can develop retinal arterial aneurysms. In 1 study of 1,007 uveitis patient charts, 14 patients $(1.39 \%)$ had macroaneurysms. ${ }^{3}$ Twelve of these patients were diagnosed with "peripheral MFC." Of these 12 patients with peripheral MFC, 5 had a definite diagnosis of sarcoidosis and 7 did not. One patient with sarcoidosis had macroaneurysms but not peripheral MFC. One patient with idiopathic uveitis had macroaneurysms. Another study ${ }^{4}$ described 48 patients with peripheral MFC; $8(17 \%)$ had arterial macroaneurysms, 3 of 8 with sarcoidosis, 1 of 5 with radiologically suggested sarcoid, 2 of 11 with laboratory evidence of sarcoid, and 2 of 24 with no evidence of sarcoid. All 8 patients with sarcoidosis were white women $>60$ years of age.

It is possible that this patient coincidentally has two distinct diseases, IRVAN and birdshot. Inflammation from birdshot may have produced the aneurysms, but this has not been previously reported. It is possible that the patient has multifocal choroidal lesions related to sarcoidosis or presumed sarcoidosis and that the aneurysms are secondary to this. In this circumstance, the HLA-A29 would be coincidental. If the choroidal lesions are clinically consistent with an MFC, most likely this represents a sarcoid-like disease with secondary retinal macroaneurysms.

\section{Dr. Emmett T. Cunningham, Jr (San Francisco, California)}

Lima and coauthors describe a fascinating 53-year-old white woman with systemic hypertension and a 20-year history of uveitis diagnosed $\sim 13$ years prior at the National Eye Institute as birdshot chorioretinopathy on the basis of the presence of mild vitreitis, numerous cream-colored choroiditis lesions, HLA-A29 antigen expression, and an otherwise negative laboratory workup. Four years after the diagnosis of birdshot chorioretinopathy, the patient developed retinal arterial macroaneurysms associated with lipid deposition and vascular changes consistent with the diagnosis of IRVAN. There seem to be two questions central to this case: first, is this birdshot chorioretinopathy and IRVAN occurring in the same patient and, if so, are there any precedents for such an occurrence? Second, if not, what else could this be?

The answer to the first question seems to be yes, at least as presented. The two groups involved in this patient's care are clearly very familiar with birdshot ${ }^{2}$ and IRVAN, $, 1,5$ including disorders known to mimic these conditions. Moreover, the sequential occurrence of two distinct uveitic syndromes has been described in other patients. Gass and Hamed ${ }^{6}$ described the first such occurrences in 1989, including a 22-year-old woman who developed acute macular neuroretinopathy followed 5 years later by the occurrence of multiple evanescent white dot syndrome (MEWDS) and a 30-year-old woman who developed MEWDS followed shortly thereafter by acute macular neuroretinopathy. Three years later, Callanan and Gass ${ }^{7}$ described a series of patients who developed MEWDS followed by MFC and two patients who developed MFC followed by MEWDS. Kozielec et $\mathrm{al}^{8}$ described the similar occurrence of MEWDS followed by MFC in a 46-year-old woman in 1991. Bryan et $\mathrm{al}^{9}$ subsequently described 4 more women aged 29 years to 34 years who developed MFC followed 5 years later by MEWDS. In 2004, Mathura et al ${ }^{10}$ described an 18-year-old man who developed acute posterior multifocal placoid pigment epitheliopathy followed 16 years later by MFC. Similarly, acute zonal occult outer retinopathy has been independently reported to occur in patients with a history of MFC, ${ }^{11}$ punctate inner choroiditis, ${ }^{12}$ and MEWDS. ${ }^{13}$ Hence, although this would be a first report of IRVAN occurring in a patient with birdshot chorioretinopathy, the patient does seem to satisfy the diagnostic criteria for the two conditions, and there are clear precedents for the sequential occurrence of two distinct uveitic syndromes in a single patient, although it has been 
suggested, at least by some of the conditions mentioned earlier forming a continuum or complex, and hence may not represent completely independent entities. ${ }^{14}$

It is important to remember, however, that neither HLA-A29 antigen expression nor choroiditis spots of the type observed in this patient are pathognomonic of birdshot chorioretinopathy. The HLA-A29 allele is present in up to $7 \%$ of whites ${ }^{2}$ and has been independently associated with idiopathic retinal vasculitis ${ }^{15,16}$ and Behçet disease. ${ }^{17}$ Moreover, choroiditis spots similar to those seen in birdshot can occur in a number of disorders, particularly sarcoidosis. ${ }^{18-20}$ In fact, at least 1 patient with known sarcoidosis and choroiditis spots has been reported to be HLA-A29-positive. ${ }^{18}$ Similarly, retinal arterial macroaneurysms are well recognized to occur in types of uveitis other than IRVAN, specifically sarcoidosis. Takagi et al ${ }^{21}$ first described arterial macroaneurysm formation in 2 patients with ocular sarcoidosis in 1994. Four years later, Rothova and Lardenoye ${ }^{3}$ reported the occurrence of arterial macroaneurysms in $17 \%$ of 48 patients with choroiditis spots, including a subgroup of patients with proven sarcoidosis. These findings were subsequently and independently confirmed by Verougstraete et al ${ }^{22}$ and Yamanaka et al, ${ }^{4}$ both of whom recommended that all patients with retinal arterial macroaneurysms and choroiditis be thoroughly evaluated for sarcoidosis. Hence, is this birdshot chorioretinopathy plus IRVAN or ocular sarcoidosis with retinal arterial aneurysm formation? If the latter, could the HLAA29 positivity have predisposed the patient to microaneurysm formation? We may never know, but an aphorism we have always found useful is that uncommon presentations of common disorders occur more frequently than common presentations of uncommon disorders. Assuming this to be true, the independent occurrence of two uncommon and unrelated disorders in the same patient would be very rare indeed. We personally would have a high index of suspicion for sarcoidosis in this patient and, if not already done, would recommend a high-resolution, contrast-enhanced computed tomographic scan of the chest, and perhaps even a gallium scan, to search for otherwise occult lymphadenopathy.

\section{Editor's Note}

Drs. Lima, Shakin, Sen, Nussenblatt, and Yannuzzi presented a 53-year-old woman with a long history of inflammation, chorioretinal lesions, disk swelling, and retinal vascular inflammatory changes and a positive HLA-A29. She was believed to have a diagnosis of birdshot chorioretinopathy. At a later time, she developed retinal arteriolar macroaneurysms with lipid exudation, neuroretinitis.

We have enlisted the help of Drs. Lee M. Jampol and Emmett T. Cunningham, Jr, to help us put this case together. Dr. Jampol reviews the differential diagnosis of retinal arterial macroaneurysms, a prominent late finding in this patient.

I. Macroaneurysms
A. Hypertension
B. Arteriosclerosis
C. Postembolic
D. Coats disease
E. Retinal vascular occlusion
F. Idiopathic polypoidal choroidal vasculopathy
G. Idiopathic retinal vasculitis, aneurysms, and neuroretinitis
H. Sarcoid 
Dr. Jampol discusses IRVAN but states that the patient's findings are not consistent with IRVAN. He notes that the lesions in this patient were consistent with birdshot chorioretinitis, but reminds us that HLA-A29 is positive in $7 \%$ of the general population. $\mathrm{He}$ notes that patients with sarcoidosis can have retinal macroaneurysms.

The fundamental question in this case has been stated by both consultants. Is this birdshot chorioretinopathy plus IRVAN or ocular sarcoidosis with retinal arterial aneurysms?

Dr. Cunningham goes through the reports of patients having two distinct uveitic syndromes but notes that there have been no reports of IRVAN occurring in a patient with birdshot choroidopathy. He states that at least one patient with sarcoidosis and choroiditis spots has been reported to be HLA-B27 positive, and patients with ocular sarcoid may have arterial macroaneurysms. With this in mind, he concludes that he has a high index of suspicion for sarcoidosis in this patient. Dr. Jampol similarly concludes that if the choroidal lesions are clinically consistent with an MFC, then this case represents a sarcoid-like disease with secondary retinal macroaneurysms.

We thank Drs. Lima, Shakin, Sen, Nussenblatt, and Yannuzzi for an outstanding case. We thank Drs. Jampol and Cunningham for their wisdom as well as their knowledge in discussing this fascinating patient.

\section{References}

1. Samuel MA, Equi RA, Chan TS, et al. Idiopathic retinitis, vasculitis, aneurysms, and neuroretinitis (IRVAN): new observations and a proposed staging system. Ophthalmology. 2007; 114:1526-1529. [PubMed: 17678691]

2. Shah KH, Levinson RD, Yu F, et al. Birdshot chorioretinopathy. Surv Ophthalmol. 2005; 50:519541. [PubMed: 16263368]

3. Rothova A, Lardenoye C. Arterial macroaneurysms in peripheral multifocal chorioretinitis associated with sarcoidosis. Ophthalmology. 1998; 105:1393-1397. [PubMed: 9709748]

4. Yamanaka E, Ohguro N, Kubota A, Yamamoto S, Nakagawa Y, Tano Y. Features of retinal arterial macroaneurysms in patients with uveitis. Br J Ophthalmol. 2004; 88:884-886. [PubMed: 15205230]

5. Chang TS, Aylward GW, Davis JL, et al. The Retinal Vasculitis Study Group. Idiopathic retinal vasculitis, aneurysms, and neuroretinitis. Ophthalmology. 1995; 102:1089-1097. [PubMed: 9121757]

6. Gass JDM, Hamed LM. Acute macular neuroretinopathy and multiple evanescent white dot syndrome in the same patients. Arch Ophthalmol. 1989; 107:189-193. [PubMed: 2916971]

7. Callanan D, Gass JDM. Multiple choroiditis and choroidal neovascularization associated with multiple evanescent white dot and acute idiopathic blind spot enlargement syndrome. Ophthalmology. 1992; 99:1678-1685. [PubMed: 1280798]

8. Kozielec GF, Wyhinny GJ, Jampol LM. Evolution of distinct chorioretinal scars in recurrent MEWDS. Retina. 2001; 21:180-182. [PubMed: 11321149]

9. Bryan RG, Freund KB, Yannuzzi LA, Spaide RF, Huang SJ, Costa DL. Multiple evanescent white dot syndrome in patients with multifocal choroiditis. Retina. 2002; 22:317-322. [PubMed: 12055465]

10. Mathura JR Jr, Jampol LM, Daily MJ. Multifocal choroiditis and acute posterior multifocal placoid pigment epitheliopathy occurring in the same patient. Arch Ophthalmol. 2004; 122:1881-1882. [PubMed: 15596597]

11. Holz FG, Kim RY, Schwartz SD, et al. Acute zonal occult outer retinopathy (AZOOR) associated with multifocal choroidopathy. Eye. 1994; 8:77-83. [PubMed: 8013724]

12. Taira K, Nakazawa M, Takano Y, Ota T. Acute zonal occult outer retinopathy in the fellow eye 5 years after presentation of punctate inner choroidopathy. Graefes Arch Clin Exp Ophthalmol. 2006; 244:880-882. [PubMed: 16315044] 
13. Fine HF, Spaide RF, Ryan EH Jr, Matsumoto Y, Yannuzzi LA. Acute zonal occult outer retinopathy in patients with multiple evanescent white dot syndrome. Arch Ophthalmol. 2009; 127:66-70. [PubMed: 19139340]

14. Gass JDM. Are acute zonal occult outer retinopathy and the white spot syndromes (AZOOR complex) specific autoimmune diseases? Am J Ophthalmol. 2003; 135:380-381. [PubMed: 12614758]

15. Bloch-Michel E, Frau E. Birdshot retinochoroidopathy and HLA-A29+ and HLA-A29- idiopathic retinal vasculitis: comparative study of 56 cases. Can J Ophthalmol. 1991; 26:361-366. [PubMed: 1764641]

16. Bergink GJ, Ooyman FM, Maas S, Rademakers AJ. Three HLA-A29 positive patients with uveitis. Acta Ophthalmol Scand. 1996; 74:81-83. [PubMed: 8689490]

17. Kaya TI, Dur H, Tursen U, Gurler A. Association of class I HLA antigens with the clinical manifestations of Turkish patients with Behçet's disease. Clin Exp Dermatol. 2002; 27:498-501. [PubMed: 12372094]

18. Vrabec TR, Augsburger JJ, Fischer DH, Belmont JB, Tashayyod D, Israel HL. Taches de bougie. Ophthalmology. 1995; 102:1712-1721. [PubMed: 9098267]

19. Read RW, Rao NA, Sharma OP. Sarcoid choroiditis initially diagnosed as birdshot choroidopathy. Sarcoidosis Vasc Diffuse Lung Dis. 2000; 17:85-86. [PubMed: 10746266]

20. Khurana RN, Parikh JG, Rao NA. Sarcoid choroiditis simulating birdshot chorioretinopathy. Retin Cases Brief Rep. 2008; 2:301-303.

21. Takagi T, Nakajima A, Sawada Y, Sakuragi S. Macroaneurysms in ocular sarcoidosis. Folia Ophthalmol Jpn. 1994; 45:555-559.

22. Verougstraete C, Snyers B, Leys A, Caspers LE. Multiple arterial ectasias in patients with sarcoidosis and uveitis. Am J Ophthalmol. 2001; 131:223-231. [PubMed: 11228299] 


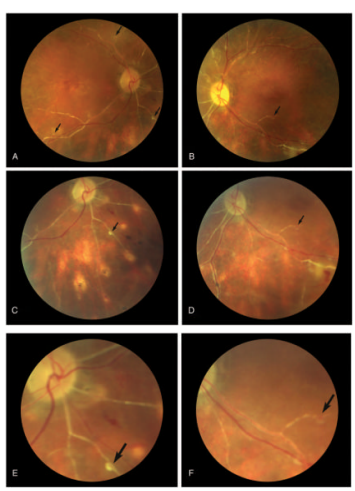

Fig. 1.

Color fundus photographs. Fundus-centered photographs of the right (A) and left (B) eyes demonstrating occlusive vasculitis and arteriolar macroaneurysm formation (arrows). Inferior fundus images of right $(\mathbf{C})$ and left eyes (D) showing arteriolar macroaneurysms (arrows) and multiple cream-colored choroidal lesions. A magnified view of the right (E) and left eyes (F) showing the arteriolar macroaneurysms (arrows). 